\title{
Particle Induced Electron Emission II
}

With Contributions by

D. Hasselkamp

H. Rothard, K.-O. Groeneveld, J. Kemmler

P. Varga, H. Winter

With 90 Figures

Springer-Verlag

Berlin Heidelberg New York

London Paris Tokyo

Hong Kong Barcelona

Budapest 


\section{Contents}

\section{Kinetic Electron Emission}

from Solid Surfaces Under Ion Bombardment

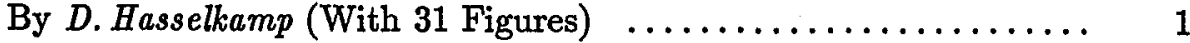

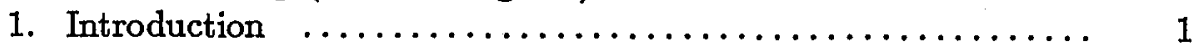

2. Definitions and Basic Quantities $\ldots \ldots \ldots \ldots \ldots \ldots \ldots \ldots, 3$

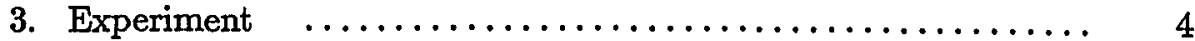

3.1 Experimental Conditions $\ldots \ldots \ldots \ldots \ldots \ldots \ldots \ldots \ldots, 4$

3.1.1 Target Preparation $\ldots \ldots \ldots \ldots \ldots \ldots \ldots \ldots \ldots .6 . \ldots$

3.1.2 Target Characterization ................. 6

3.2 Measurement of the Total Electron Yield ........... 8

3.2.1 The Quotient Method ................... 8

3.2.2 The Ion-Electron-Converter ............... 12

3.3 Measurement of Energy Spectra of Emitted Electrons ... 14

3.4 Measurement of the Angular Distribution ............ 18

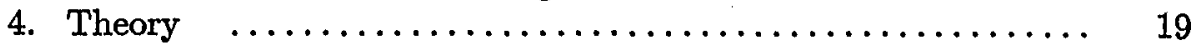

4.1 General Considerations .................... 19

4.1.1 Generation of Secondary Electrons .......... 19

4.1.2 The Diffusion Process .................. 21

4.1.3 Penetration of the Surface Barrier .......... 22

4.2 Summary of Main Theoretical Results ............. 24

4.3 Special Effects in Electron Emission $\ldots \ldots \ldots \ldots \ldots \ldots .30$

4.3.1 Recoil Ionization $\ldots \ldots \ldots \ldots \ldots \ldots \ldots \ldots \ldots . \quad 30$

4.3.2 One-Electron Plasmon Decay $\ldots \ldots \ldots \ldots \ldots \ldots .31$

4.3.3 Electron Loss from the Projectile ........... 32

4.3.4 The Molecular Effect $\ldots \ldots \ldots \ldots \ldots \ldots \ldots \ldots, 33$

4.3.5 Auger-Electron Emission ................ 35

4.3.6 The Binary Encounter Peak $\ldots \ldots \ldots \ldots \ldots \ldots . \quad 35$

5. Summary of Typical Results $\ldots \ldots \ldots \ldots \ldots \ldots \ldots \ldots \ldots . \quad 36$

5.1 Summary of Experimental Work

in the Period from 1968 to $1990 \ldots \ldots \ldots \ldots \ldots \ldots \ldots .36$

5.2 Energy Dependence of Total Yields from Metal Targets at Normal Incidence $\ldots \ldots \ldots \ldots \ldots \ldots \ldots \ldots \ldots . \ldots \ldots$

5.2.1 Positive Ion Impact .................. 42

5.2.2 Relation of Yields to the Inelastic Stopping Power 44

5.2.3 Impact by Neutral and Negative Particles ...... 48

5.2.4 Impact by Molecular Projectiles ............ 51

5.2 .5 Cluster Impact $\ldots \ldots \ldots \ldots \ldots \ldots \ldots \ldots \ldots \ldots, \quad 53$ 
5.3 Dependence of the Total Yields

on the Projectile-Target-Combination $\ldots \ldots \ldots \ldots \ldots .53$

5.4 The Total Yield as a Function of the Angle of Incidence . 56

5.5 The Angular Distribution of Emitted Electrons ........ 58

5.6 Energy Spectra of Emitted Electrons from Clean Metal Surfaces $\ldots \ldots \ldots \ldots \ldots \ldots \ldots \ldots . \ldots$

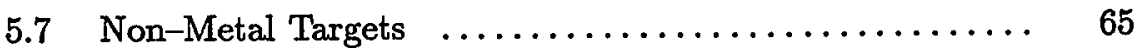

5.7.1 Semi-Conducting Materials $\ldots \ldots \ldots \ldots \ldots \ldots \ldots .65$

5.7.2 Insulating Targets $\ldots \ldots \ldots \ldots \ldots \ldots \ldots \ldots, 66$

5.8 Influence of Surface Layers $\ldots \ldots \ldots \ldots \ldots \ldots \ldots \ldots \ldots, 69$

5.9 Impact by Multiply-Charged Ions $\ldots \ldots \ldots \ldots \ldots \ldots \ldots, 71$

5.10 Temperature Dependence $\ldots \ldots \ldots \ldots \ldots \ldots \ldots \ldots \ldots .74$

5.11 Emission of Polarized Electrons $\ldots \ldots \ldots \ldots \ldots \ldots \ldots . \quad 74$

6. Applied Aspects of Ion-Induced Electron Emission $\ldots \ldots \ldots . \quad 75$

7. Summary and Outlook $\ldots \ldots \ldots \ldots \ldots \ldots \ldots \ldots \ldots \ldots, \quad 80$

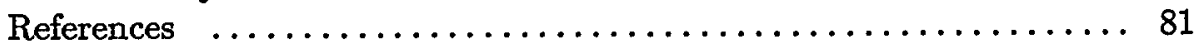

Kinetic Electron Emission from Ion Penetration of Thin Foils in Relation to the Pre-Equilibrium of Charge Distributions By H. Rothard, K.O. Groeneveld and J. Kemmler

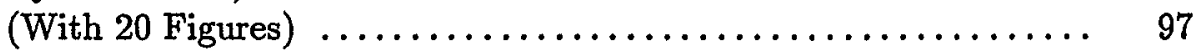

1. Introduction $\ldots \ldots \ldots \ldots \ldots \ldots \ldots \ldots \ldots \ldots \ldots \ldots \ldots \ldots \ldots \ldots, 97$

2. Forward and Backward Electron Yields in the Charge Equilibrium $\ldots \ldots \ldots \ldots \ldots \ldots \ldots \ldots . \quad 99$

2.1 Proportionality Between Secondary Electron Yields and Stopping Power $\ldots \ldots \ldots \ldots \ldots \ldots \ldots \ldots \ldots . \ldots \ldots$

2.2 Forward to Backward Secondary Electron Yield Ratio ... 104

2.3 Projectile Dependence of Electron Yields and the Relation to the Effective Near-Surface Energy Loss

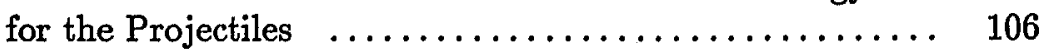

3. Electron Energy and Angular Distributions

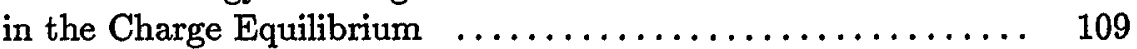

3.1 Electron Spectra as a Function of the Emission Angle ... 109

3.2 Electron Angular Distributions: Refraction of Electrons . 113

3.3 Dependence of Electron Spectra on the Surface Coverage with Adsorbates $\ldots \ldots \ldots \ldots \ldots, 116$

3.4 Molecular Effects ....................... 120

4. Pre-Equilibrium Electron Yields $\ldots \ldots \ldots \ldots \ldots \ldots \ldots \ldots, 122$

4.1 Electron Yields as a Probe of Pre-Equilibrium Stopping Power $\ldots \ldots \ldots \ldots \ldots \ldots, 122$

4.2 Electron Emission from Molecular Ion Impact ........ 126

5. Pre-Equilibrium Electron Spectra: Convoy Electrons $\ldots \ldots \ldots . \quad 129$

5.1 The Four-Step-Model for Electron Emission:

Preparation-Production-Transport-Transmission ....... 130

5.2 Application to Convoy Electron Emission ........... 134 
6. Conclusion $\ldots \ldots \ldots \ldots \ldots \ldots \ldots \ldots \ldots \ldots \ldots \ldots \ldots, 141$

References $\ldots \ldots \ldots \ldots \ldots \ldots \ldots \ldots \ldots \ldots \ldots \ldots \ldots \ldots \ldots . \ldots \ldots, 142$

Slow Particle-Induced Electron Emission from Solid Surfaces

By $P$. Varga and $H$. Winter (With 39 Figures) $\ldots \ldots \ldots \ldots \ldots . \ldots 149$

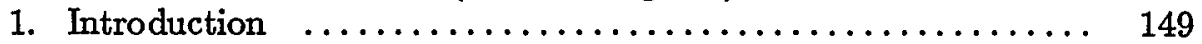

2. Experimental Techniques for Investigation of sPIE $\ldots \ldots \ldots \ldots \quad 151$

2.1 Preparation of Slow Particle Beams ............... 152

2.2 Target Preparation $\ldots \ldots \ldots \ldots \ldots \ldots \ldots \ldots \ldots \ldots .154$

2.3 Detection of Reaction Products $\ldots \ldots \ldots \ldots \ldots \ldots \ldots .155$

2.4 Measurement of Electron Emission Yields .......... 155

2.5 Measurement of Ejected Electron Energy Distributions .. 156

2.6 Measurement of Electron Emission Statistics ......... 157

3. Review on Potential Electron Emission Processes .......... 157

3.1 Models for Potential Electron Emission ............ 158

3.2 Experimental Results on PE - Total Electron Yields .... 166

3.2.1 Singly Charged Projectiles ............... 167

3.2.2 Excited Neutral Projectiles (Metastable Atoms) .. 169

3.2.3 Doubly Charged Projectiles ............... 169

3.2.4 Multicharged Projectiles $\ldots . \ldots \ldots \ldots \ldots \ldots . . .171$

3.3 Experimental Results on PE -

Electron Energy Distribution $\ldots \ldots \ldots \ldots \ldots \ldots \ldots \ldots .175$

3.3.1 Impact of Metastable Atoms .............. 175

3.3.2 Impact of Ions $\ldots \ldots \ldots \ldots \ldots \ldots \ldots \ldots \ldots, 176$

3.4 Experimental Results on PE -

Electron Emission Statistics .................. 187

4. Slow Particle-Induced Electron Emission

at the Kinetic Threshold $\ldots \ldots \ldots \ldots \ldots \ldots \ldots \ldots \ldots \ldots, 196$

4.1 The Precise Threshold of Kinetic Emission ........... 197

4.2 On the Interdependence of PE and KE ........... 198

4.3 Effects of Projectile Shielding

in Slow Particle-Induced Kinetic Emission ......... 201

5. Conclusions and Outlook $\ldots \ldots \ldots \ldots \ldots \ldots \ldots \ldots \ldots \ldots, 208$

References $\ldots \ldots \ldots \ldots \ldots \ldots \ldots \ldots \ldots \ldots \ldots \ldots \ldots \ldots \ldots . \ldots \ldots, 209$

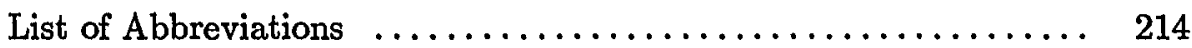

Subject Index $\ldots \ldots \ldots \ldots \ldots \ldots \ldots \ldots \ldots \ldots \ldots \ldots \ldots \ldots, 215$ 